\title{
Olea europaea and its Constituents Promote Bone Health by Enhancing Osteoblast Differentiation and Proliferation: A Review
}

\author{
Ruszymah Bt Hj Idrus ${ }^{1, *}$, Aminuddin Bin Saim²
}

\section{Ruszymah Bt Hj Idrus ${ }^{1, *}$, Aminuddin Bin Saim ${ }^{2}$}

\section{${ }^{1}$ Department of Physiology, Universiti Kebangsaan Malaysia Medical Centre, Cheras, 56000, MALAYSIA. \\ ${ }^{2}$ Ear, Nose and Throat Consultant Clinic, Ampang Puteri Specialist Hospital, Ampang, 68000, MALAYSIA. \\ Correspondence \\ Prof. Ruszymah Bt Hj Idrus \\ Department of Physiology, Universiti Kebangsaan Malaysia Medical Centre, Cheras, 56000, MALAYSIA \\ Phone no: +60391458617 \\ E-mail: ruszyidrus@gmail.com \\ History \\ - Submission Date: 17-08-2018 \\ - Review completed: 19-09-2018; \\ - Accepted Date: 15-11-2018}

DOI : 10.5530/pj.2019.1.1

Article Available online

http://www.phcogj.com/v11/i1

\section{Copyright}

(C) 2019 Phcog.Net. This is an openaccess article distributed under the terms of the Creative Commons Attribution 4.0 International license.

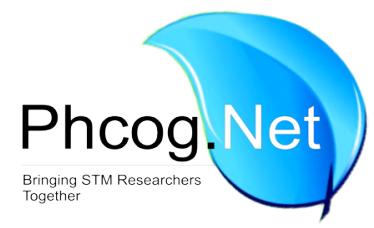

\begin{abstract}
Background: The human bone is in a constant state of balance between bone resorption and bone formation, maintained by the osteoclasts and osteoblasts respectively. Association of Mediterranean diet and bone health has been prevalently studied in recent years. Olive and its constituents have been suggested to be the major contributor to the benefits of the Mediterranean diet in bone health. Method: In this review, cellular and molecular mechanism of bone homeostasis and the influence of olive and its constituents were discussed. Result: Many studies ranging from clinical, animal and in vitro cell culture reported benefits of olive and its constituents in bone health. Olive and its constituent exerted its beneficial effect in bone health through stimulation of bone formation as opposed to inhibition of bone resorption Conclusion: Olive and its constituents promotes osteoblast differentiation and proliferation. Key words: Bone, Olive, Oleuropein, Hydroxytyrosol, Osteoblast.
\end{abstract}

\section{INTRODUCTION}

The bone structure and the cellular processes that occur within the bone, allows it to simultaneously serve as a calcium reservoir while providing structure support and locomotion. As a result, the human bone is in a constant state of balance, between deposition and resorption of bone minerals to maintain the calcium homeostasis and bone integrity. ${ }^{1}$ Bone minerals is deposited by osteoblasts in a process called ossification and is resorbed by osteoclasts to meet physiological calcium demand in the body. ${ }^{2}$

Condition that result in a decrease in bone mass, such as osteoporosis, can either be caused by a decrease in ossification or an increase in resorption. ${ }^{3}$ The ossification of bone involves a complex series of events that encompasses osteoblasts proliferation and differentiation. ${ }^{4}$ Alternatively, the bone reabsorption process involves the formation and activation of osteoclasts, which is derived from monocytes or macrophages. ${ }^{5}$

Physiologically, bone modelling and remodeling processes are governed not only by heritable traits but also by nutritional, mechanical and hormonal factors. Modification towards dietary patterns has been associated with reduced risk in osteoporosis incidence. ${ }^{6} \mathrm{~A}$ group that studied the osteoporosis incidence among Greece women noted that the osteoporosis incidence in their population is substantially lower than in the US and other European countries. They hypothesized that Mediterranean diet to play a major role in this difference. ${ }^{7}$

The Mediterranean diet is defined by high intake of olive oil, plant products, fish and seafood; a low intake of dairies, meat and meat products; and a moderate ethanol intake. ${ }^{8}$ These food items possessed a complex array of naturally occurring bioactive molecules with antioxidant, anti-inflammatory and alkalizing properties that may contribute to the bone-sparing effect of the Mediterranean diet. ${ }^{6}$ Adherence to the Mediterranean diet has been linked to the prevention of bone diseases via a number of observational studies done across different populations. , $9,10^{2}$

Upon inspection of the studies that associated Mediterranean diet with bone health, olive oil has been considered to be the major contributor to the health benefits associated with this diet. ${ }^{6}$ Olive oil is the major source of fats in the Mediterranean diet. Olive oil has been associated with reduction in the incidence of some diseases such as cardiovascular, diabetes and several cancers, among other degenerative pathologies. ${ }^{11}$

Some health properties of olive-tree-derived products have been attributed to its phenolic compound, oleuropein. Oleuropein is a key olive-tree phenolic compound with high antioxidant capacity. ${ }^{12}$ Therefore, it has interesting health effects, including anti-inflammatory, anti-atherogenic, anti-tumoral, anti-microbial and neuroprotective. They can be found in various parts of the olive tree plant, like fruits and leaves, as well as mill by-products of olive-oil production, including waste water. ${ }^{13}$

Olive, olive oil and its bioactive component has been nominated as a potential candidate for dietary options against osteoporosis. This is based on the growing body of literature focusing on the bone protective effects of olive and its constituents. ${ }^{14}$ 
Parallel to the growing body of literature on bone protective effects of olive and its constituents, understanding of its mechanism of action at the molecular level should follow. Hence, this review seeks to elucidate the molecular mechanism utilized by olive and its constituent in promoting bone health.

\section{Cellular Physiology of Bone Homeostasis}

At the hormonal level, calcium homeostasis is regulated by the parathyroid hormone. When the blood calcium level is low, production of parathyroid hormone triggers the physiologic response that leads to the release of bone calcium, increase of intestinal calcium absorption and increase of renal calcium reabsorption. All in the effort of increasing the calcium level back to the normal physiologic level. ${ }^{15}$

Within the bone, bone homeostasis is a dynamic process that involves the interaction between two distinct bone cells, osteoblasts and osteoclasts. It is the balance between the formation of bone and its resorption, executed by the osteoblasts and osteoclasts respectively. ${ }^{16}$

Osteoblast, the cell that is responsible for new bone formation, is derived from osteoprogenitors, the undifferentiated pluripotent mesenchymal cells that are responsible for bone development. ${ }^{17}$ Osteoblast differentiation is controlled by the master transcription factor RUNX2 (runt-related transcription factor 2); also known as CBFA1 (core-binding factor A1). ${ }^{18}$ Osterix (Osx), is another transcription factor that is also important in osteoblast differentiation. ${ }^{19}$ Studies with mice that lack either of these proteins demonstrated the formation of a cartilaginous skeleton at embryonic level but not the bony skeleton, resulting in the morbidity of the mice at birth. ${ }^{20}$

Osteoblasts synthesized collagen and two specialized proteins, osteocalcin and osteopontin. ${ }^{21}$ Together, the latter two protein is known as osteoid. Osteoblasts also produce alkaline phosphatase, the enzyme that is required to form the calcium phosphate crystals, hydroxyapatite. ${ }^{2}$ This will eventually lead to the formation of mineralized extracellular matrix that made up the structure of the bone.

As mineralization occurs, some osteoblasts will mature into osteocytes and occupy the lacunae, the hollow spaces within the bone, to maintain its structure. Once mineralization is done, active osteoblasts will become flattened and line the surface of the bone. These mature osteoblasts are known as the lining cells. The rest of the osteoblast simply undergo apoptosis. ${ }^{1}$

Osteocytes form a network extending throughout mineralized bone via its long, dendrite-like processes, in order to interact with other osteocytes within the mineralized bone and the osteoblasts on the bone surface. ${ }^{22}$ Osteocytes respond to mechanical load and this network is thought to be integral in the detection of mechanical strain and associated bone microdamage that accumulates as a result of normal skeletal loading and fatigue. $^{22}$

On the other hand, the bone resorption involves osteoclasts, which is derived from monocytes or macrophages. ${ }^{5}$ Osteoclast is an irregular shaped, giant cell, that break down or reabsorbs the bone. Osteoclasts arise from either macrophages or monocytes from the bone marrow. Monocytes fuse together to form multinucleated osteoclasts cells. ${ }^{23}$

Osteoclast cells have numerous fingerlike processes known as the ruffled border. The ruffled border is the side where osteoclast attached to the surface of the bone, maximizing the surface area for the resorption of the bone minerals. Osteoclasts bind themselves to the bone via the integrin protein, vitronectin. The site where the osteoclast attached to the bone is known as Howship's lacunae, commemorating the biologist John Howship that first discover it. Osteoclasts secrete tartrate resistant acid phosphates (TRAP) in order to lowers the $\mathrm{pH}$ level in the Howship's lacunae to aid the reabsorption of the mineralized bone matrix. ${ }^{24}$
Cathepsin $\mathrm{K}$ is a cysteine protease enzyme present in actively resorbing osteoclasts. It is able to cleave the telopeptide region of type 1 collagen allowing for the mineralized bone resorption by the osteoclast. The serum level of cathepsin $\mathrm{K}$ reflects the number of osteoclasts and serves as a specific biomarker of osteoclast activity. ${ }^{24}$

The activation of osteoclasts is known to be regulated by two cytokines; receptor activator of nuclear factor- $\kappa \beta$ ligand (RANKL) and macrophage colony-stimulation factor (M-CSF). The binding of RANKL to its receptor RANK on the surface of osteoclast, leads to the activation of TNF receptorassociated factor 6 (TRAF6), which is linked to nuclear factor $\kappa B$ $(\mathrm{NF}-\kappa \mathrm{B})$ via mitogen-activated protein kinases (MAPKs). RANKL and $\mathrm{M}$-CSF are proteins secreted by osteoblasts and is important for the formation of osteoclast and regulation of its activity. ${ }^{25}$

Osteoblasts also secretes osteoprotegerin (OPG), a soluble decoy receptor for RANKL that blocks its binding to RANK. ${ }^{25}$ The OPG secretion by osteoblast prevents osteoclast differentiation, fusion and activation, which causes a decrease in bone reabsorption and destruction. ${ }^{25}$ Secretion of transforming growth factor $\beta$ (TGF- $\beta$ ) by osteocytes, induced the secretion of OPG, resulting in the inhibition of osteoclastogenesis. ${ }^{26}$

\section{Markers of Bone Health}

For bone health assessment and evaluation of therapeutic responses, a number of markers have been used, not just for clinical tests but also applicable to experimental studies using in vitro (cell-based) assays or in vivo (animal) models. Two categories of bone parameters can be measured to evaluate bone health, which is the physical and biochemical markers. $^{27}$

A major physical parameters of bone health is the bone mineral density (BMD). BMD is the measurement of the amount of calcified tissue in the bone. BMD can be obtained using X-rays. The procedure is painless and non-invasive and involves low radiation exposure. For small animals, mini X-rays equipment is often used. As the prime indicator of bone strength, BMD has become the standard for diagnosis of osteoporosis as recommended by the World Health Organization. However, while BMD is a quantitative assessment of bone health, it does not provide information on bone quality. ${ }^{28}$

$\mathrm{BMD}$ results can be supplemented with the measurement of the bone architecture to get an overall picture of bone quality. ${ }^{29}$ Bone microarchitecture is accessed via histological observation. Bone microarchitecture is related to the mechanical strength. Deterioration of bone architecture results in bone loss and can be linked to observations such as decreased number of trabeculae, increased inter-trabecular distances, loose connectivity of the trabecular meshwork, reduction of cortical bone thickness and increased porosity. Apart from BMD measurement and bone microstructure assessment, there are plethora of other less common method to measure bone strength and fragility employed in animal models to test for drug effects. ${ }^{30}$

The use of bone biochemical markers to measure and monitor bone turnover and bone loss is considered recent, thanks to the in-depth understanding of the bone physiology from recent years. ${ }^{31}$ The markers reflect the metabolic activity of osteoblasts or osteoclasts and are measurable in blood or urine in order to provide a quantitative estimate of the status of bone remodeling.

Information on bone remodeling status is an early indicator of pathological changes or the risk of some bone diseases. These biomarkers are useful not only for clinical assessments as monitors of osteoporosis and predictors of fracture, but also for the evaluation of therapeutic responses in experimental model.

In terms of evaluating therapeutics responses to novel drugs or natural products, bone biomarkers provide immediate information as compare 
to measuring the physical bone deposition, which could take some time before significant changes can be detected. Bone biomarkers can be categorized into two, the bone formation markers and the bone resorption markers.

Biomarkers for bone formation are by-products or enzymes used by the active osteoblast during bone formation. Among products of bone formation that can be used as biochemical markers are alkaline phosphatase, osteocalcin and the pro-collagen type 1. Increase in genetic or protein expression of transcription factors that regulate osteogenesis, RUNX2 and Osterix are also useful in measuring bone formation.

In contrast, biomarkers for bone resorption will be the products associated with the activity of osteoclast. Among them are TRAP, RANKL, cathepsin $\mathrm{K}$, osteopontin and hydroxproline (component of collagen).

\section{Olea europaea and Its Component}

Olive or its scientific name, Olea europaea is the fruit from the olive tree, which belongs to the family Oleaceae. It is one of the major components in the diet of Mediterranean people. A closer inspection on the health benefits of the olive oil alone suggested that it is the major contributor to most of the health benefits associated with Mediterranean diet. In the review, clinical and epidemiological data that looks into the relevance of olive oil and its components for longevity and against age- and lifestyleassociated pathologies such as cancer, cardiovascular, metabolic and neurodegenerative diseases correlate well with the positive outcomes brought by the Mediterranean diet. ${ }^{11}$

The promising potential of olive health benefits is due to the presence of bioactive phenolics compounds. The major phenolic compounds in olive oil can be divided into three different classes: simple phenols (hydroxytyrosol, tyrosol); secoiridoids (oleuropein, the aglycone of ligstroside and their respective decarboxylated dialdehyde derivatives) and lignans (1-acetoxypinoresinol and pinoresinol). ${ }^{32}$ Phenolic compounds of olive contributed to the antioxidative properties of olive.

Traditionally, olive has been used in treating asthma, hemorrhoids, intestinal diseases and use to reduce blood sugar and cholesterol level. Recent human and animal research have shown the importance of olive oil in reducing the incidence of some diseases such as cardiovascular, diabetes and several cancers, among other degenerative pathologies. ${ }^{11}$ In terms of bone health, its association with Mediterranean diet has been prevalently studied in recent years. Olive and its constituents have been suggested to be the major contributor to the benefits of the Mediterranean diet in bone health.

\section{Clinical Studies Reporting Effect of Olea europaea and its Constituents in Osteoporosis Prevention}

Among the risk factors that can influence osteoporosis, dietary consumption is the factor that can be modified. Hence, researchers are motivated to find the link between dietary consumption and osteoporosis. To date, the most consistently followed approach to examine the potential relation between dietary factors and skeletal health was based on particular micronutrients such as calcium and vitamin D3. ${ }^{33}$ However, in reality, people do not eat isolated nutrients but meals consisting of a variety of foods with complex combinations of nutrients and bioactive components. ${ }^{6}$

One of the potential candidates is the Mediterranean diet. The Mediterranean diet is defined by a high intake of olive oil, plant products, fish and seafood; a low intake of dairies, meat and meat products; and a moderate ethanol intake. ${ }^{8}$ It has been noted that several studies conducted in the Mediterranean area have reported osteoporosis and osteoporosis-related fractures incidences that is lower among countries across the European Union. ${ }^{7,10,34}$ This brings about the interest on linking the Mediterranean diet consumption and osteoporosis prevention.
Adherence to the Mediterranean diet has been shown to reduce rate the hip fracture incidence in Swedish men and women. ${ }^{34}$ The study combined two Swedish cohort studies consisting of 37,903 men and 33,403 women (total $n=71,333$, mean age 60 years) that are free of previous cardiovascular disease and cancer who answered a medical and a food-frequency questionnaire in 1997. A modified Mediterranean diet score (mMED; range 0-8 points) was created based on high consumption of fruits and vegetables, legumes and nuts, whole grains, fermented dairy products, fish and olive/rapeseed oil, moderate intake of alcohol and low intake of red and processed meat. Incident hip fractures between 1 January 1998 and 31 December 2012 were retrieved from the National Patient Register and the result shows an inverse relationship between the higher Mediterranean diet score and low incidence rate in hip fracture.

In the clinical trial PREDIMED, 127 elderly men were divided into three groups, Mediterranean diet with virgin olive oil (at least $50 \mathrm{~mL} /$ day), Mediterranean diet with mixed nuts (at least $30 \mathrm{~g} /$ day) or a low-fat diet and were followed-up for two years. The virgin olive oil group demonstrated an increased in bone formation marker and decreased in bone resorption markers, suggesting the positive effects that olive have in bone remodeling. ${ }^{10}$

A group of researchers in Italy investigated the effect of 1 year of oral supplementation with either extra virgin olive oil (VOO) enriched with vitamins D3, K1 and B6 (VitVOO) or VOO used as placebo (PlaVOO) on modification of bone turnover and oxidative stress markers. ${ }^{35}$ The study revealed that subjects taking VitVOO showed lower plasma osteocalcin levels than those taking the PlaVOO. In terms of bone mineral density, a significant improvement in VitVOO subjects compared to PlaVOO was found. All oxidative stress markers as thiobarbituric acid reactive substances, lipid hydroperoxides and conjugated dienes showed a significant reduction after VitVOO supplementation, whilst plasma total antioxidant capacity values were significantly increased in VitVOO group compared to PlaVOO group. Although all the findings in the study demonstrate the beneficial effect of vitamin on bone health markers compare to the virgin olive oil control, the fact that the placebo resulted in favorable outcome. This is in agreement with the beneficial effects of VOO documented in other studies. ${ }^{36-38}$

\section{Animal Studies Reporting Effect of Olea europaea and its Constituents in Osteoporosis Prevention}

In terms of olives fruit, its inclusion in the diet of rat's model of senile osteoporosis, prevented bone loss in the whole femur and at cortical sites of the animals. ${ }^{39}$ However, this effect is only seen in the black olives but not green olive, a more common variant of olive. This could be attributed to a higher polyphenol level in the black olives compared to the green olives.

Estrogen, a reproductive hormone has long been associated with osteoporosis. Decreased estrogen predisposed to increased risk of osteoporosis. When discussing the olive oil, it has been associated with prevention of the bone loss caused by the absence of estrogen in ovariectomized rats. ${ }^{40-42}$ The effect is associated with olive oil anti-oxidant properties as supported by the decrease in oxidative markers in the serum. ${ }^{41}$ The comparison between olive oil supplementation and the synthetic estrogen, suggested the efficacy of olive oil as the dietary alternative to estrogen replacement in preventing post-menopause bone loss. ${ }^{40}$

Positive effect of olive oil in improving bone density can be observed when Liu et al. (2014) analyzed the blood samples from patients who regularly consumed olive oil over a year. The bone mineral density of the lumbar spine and left femur of the patients were evaluated by dual energy X-ray absorptiometry and positive correlation between olive oil consumption and bone mineral density was evident. ${ }^{40}$ 


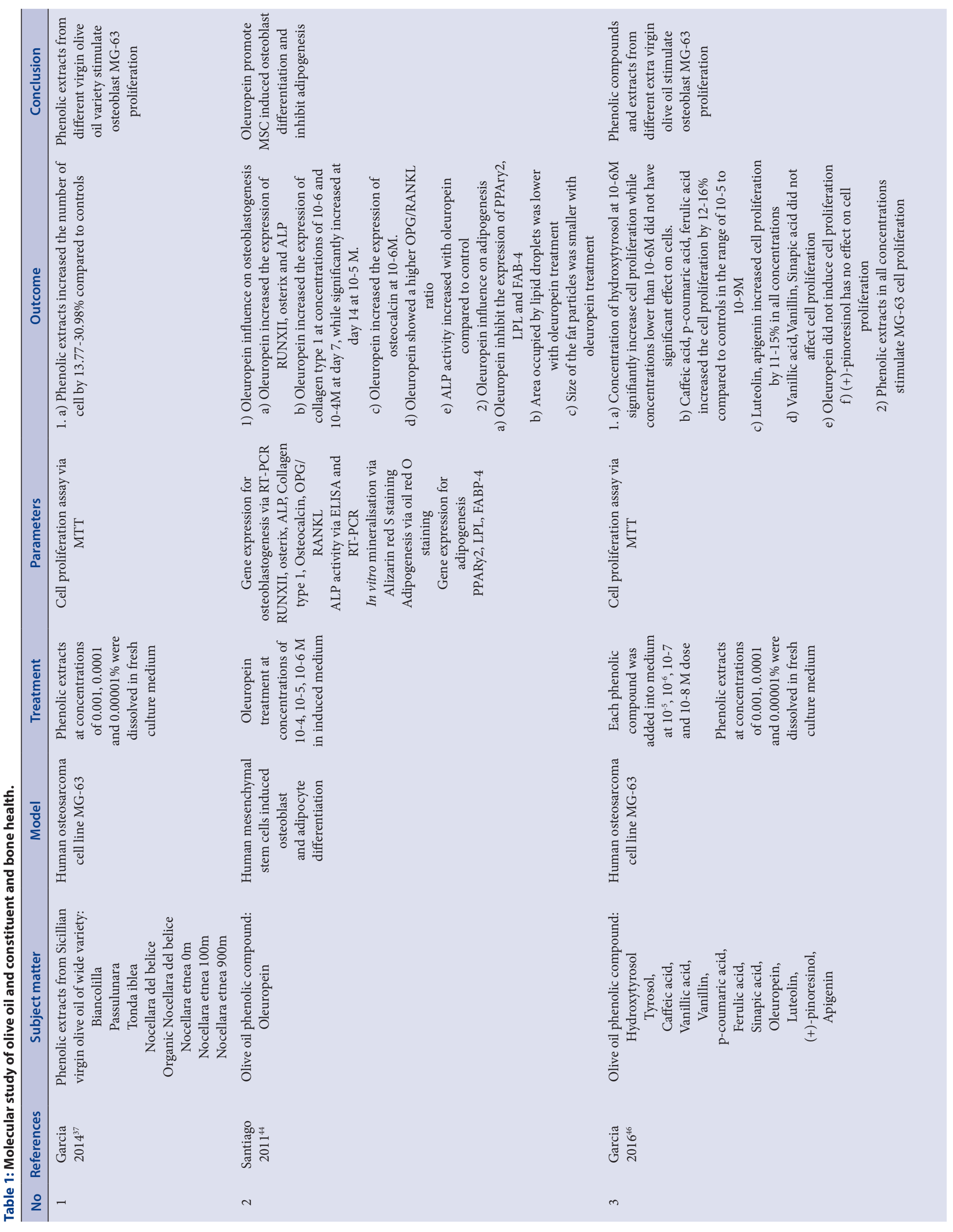




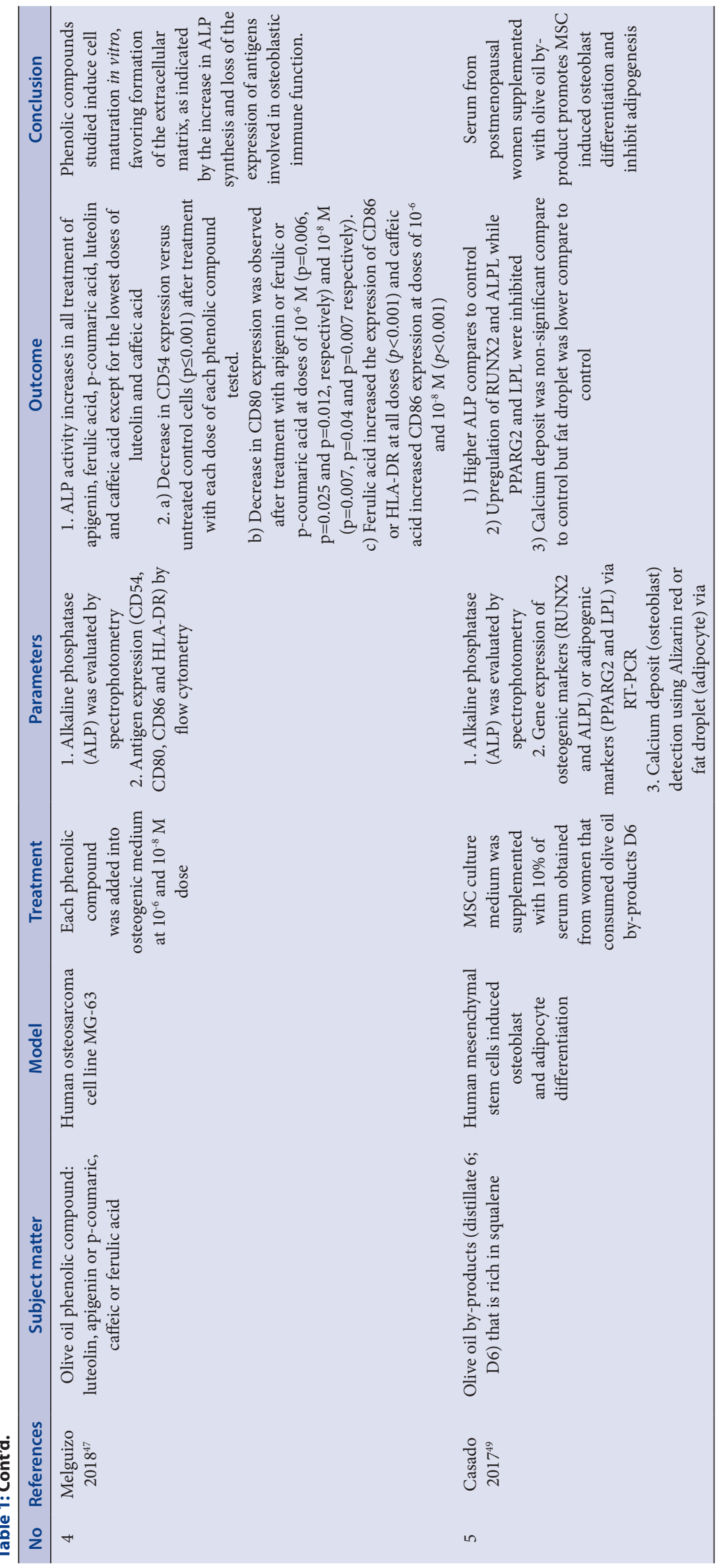


The correlation between olive oil antioxidant properties and bone remodeling can be observed when osteoporosis patients, supplemented with extra virgin olive oil diet, presented an increased in bone formation marker and decreased in bone resorption markers, together with the increased in antioxidant capacity and decreased levels of oxidative products. $^{35}$

Oleuropein when given to inflamed ovariectomized rats, prevented the decline of total, diaphyseal and metaphyseal bone mineral density. This is due the attenuation of bone resorption, indicated by the decrease of serum osteocalcin and a marginal decrease of urinary deoxypyridinoline level. ${ }^{43}$

Taken together, the bone protective effects of olive and its derivative is well established via clinical and pre-clinical model. Hence, elucidating the intracellular molecular signaling involved in the olive effects on both osteoblasts and osteoclasts is of paramount importance.

\section{In vitro Studies Reporting Effect of Olea europaea and its Constituents in Osteoporosis Prevention}

From the literature review, 5 studies that investigated the effect of olive and its constituent on in vitro culture of human cells has been identified and listed in Table 1. Two studies used bone marrow derived mesenchymal cell to investigate the effect that oleuropein ${ }^{44}$ and olive oil by-products ${ }^{45}$ have on osteoblast differentiation. Another three studies, used human osteosarcoma cell line MG-63 to investigate the effect of olive oil phenolic compounds on the cell proliferation of the osteoblast ${ }^{37,46}$ and expression of bone differentiation markers. ${ }^{47}$

Bone formation is dependent on the cell proliferation of the osteoblast. Evaluation of olive oil and its constituent was reported by Garcia et al. In the earlier study, they reported significant increase in proliferation with phenolic extract from a number of Sicillian virgin olive oil variants. ${ }^{37}$ In a more recent study, they supplemented the osteoblast cell with both extra virgin olive oil extracts and isolated phenolic compounds namely hydroxytyrosol, tyrosol, caffeic acid, vanillic acid, p-coumaric, ferulic acid, sinapic acid, oleuropein, luteolin, pinoresinol and apigenin. They also reported stimulation of the osteoblast proliferation with phenolic compounds and extracts. ${ }^{46}$ The authors suggested that the beneficial effect of olive oil and its constituent on the proliferation of osteoblast in culture is mainly due to its anti-oxidant effect.

In bone marrow derived mesenchymal cell culture, oleuropein has been reported to enhance osteoblastogenesis but suppress adipogenesis. ${ }^{44}$ This suggested that oleuropein play a role in the lineage specific differentiation of bone marrow stem cell. Oleuropein-treated cells demonstrated increased expression of osteoblastogenesis genes such as runt-related transcription factor II, osterix, collagen Type I, alkaline phosphatase and osteocalcin. ${ }^{44}$ The role of olive phenolic compound in selective induction of osteoblastogenesis is supported by the experiment using serum from postmenopausal women supplemented with olive oil by-product. The diet of post-menopausal women was supplemented with olive oil distillate that is high in squalene, a component of olive oil, for two months and serum was collected. Treatment of human bone marrow-derived mesenchymal stem cell with the serum demonstrated an induction of osteogenesis and suppression of adipogenesis. ${ }^{48}$

Role of olive oil phenolic compound in osteoblast differentiation was also reported in human osteosarcoma cell line MG-63. Different phenolic compounds of olive oil which are luteolin, apigenin, $\mathrm{p}$-coumaric, caffeic acid and ferulic acid were supplemented to the bone cell culture to investigate the expression of bone formation and immunological markers. All phenolic compounds tested were able to induce cell maturation in vitro as indicated by the increase in ALP synthesis and loss of the expression of antigens involved in osteoblastic immune function. ${ }^{47}$ This suggested that olive and its constituent beneficial effect on bone health by inducing bone formation while simultaneously inhibiting bone resorption process.

\section{CONCLUSION}

The literature review reveals numerous evidences supporting the role of olive and its constituents on bone health. The evidence is available in a wide spectrum of experimental studies, including in vitro cell culture, animal models and clinical studies. Knowledge on the molecular mechanism of bone homeostasis has been well established, enabling scientist to understand the influence of olive and its constituents on these mechanism of action at the molecular level. Olive and its constituents seem to exert beneficial effect on the bone homeostasis via inducing the bone formation process as opposed to the inhibition of bone resorption process. In particular, olive and its constituent has been shown to promote osteoblast differentiation and proliferation.

\section{ACKNOWLEDGEMENT}

We would like to thank the Faculty of Medicine UKM for providing resources to write this review.

\section{CONFLICT OF INTEREST}

The authors declare no conflict of interest.

\section{ABBREVIATIONS}

RUNX2: Runt-related transcription factor 2; CBFA1: Core binding factor A1; Osx: Osterix; TRAP: Tartrate resistant acid phosphatase; RANKL: Receptor activator of nuclear factor- $\kappa \beta$ ligand; M-CSF: Macrophage colony-stimulation factor; TRAF6: TNF receptor-associated factor 6; NF-к $\beta$ : Nuclear factor $\kappa \beta$; MAPK: Mitogen-activated protein kinase; OPG: Osteoprotegerin; TGF- $\beta$ : Transforming growth factor $\beta$; BMD: Bone mineral density; mMED: Modified Mediterranean diet score.

\section{REFERENCES}

1. Clarke B. Normal bone anatomy and physiology. Clin J Am Soc Nephrol. 2008;3(Suppl 3):131-9

2. Florencio-Silva R, Sasso GRDS, Sasso-Cerri E, Simões MJ, Cerri PS. Biology of bone tissue: Structure, function and factors that influence bone cells. Biomed Res Int. 2015;Article ID 421746.

3. Dolzhenko AT, Sagalovsky S. Cellular and molecular mechanisms of osteoporosis: Current concepts and future direction treatment. Sovrem Revmatol. 2016;10(2):56-63.

4. Arvidson K, Abdallah BM, Applegate LA, Baldini N, Cenni E, Gomez-Barrena E et al. Bone regeneration and stem cells. J Cell Mol Med. 2011;15(4):718-46.

5. Xu F, Teitelbaum SL. Osteoclasts: New insights. Bone Res. 2013;1(1):11-26

6. Pérez AR, Velasco AR. Adherence to Mediterranean diet and bone health. Nutr Hosp. 2014;29(5):989-96.

7. Trichopoulou A, Georgiou E, Bassiakos Y, Lipworth L, Lagiou P, Proukakis C, et al. Energy Intake and Monounsaturated Fat in Relation to Bone Mineral Density among Women and Men in Greece 1. Prev Med (Baltim). 1997;26(3):395-400.

8. Arós F, Corella D, Covas MI, Estruch R, Fiol M, Lapetra J, et al. How to publish in The New England Journal of Medicine and not to die while trying it: The PREDIMED experience. Nutr Hosp. 2013;28(4):977-9.

9. Savanelli MC, Barrea L, Macchia PE, Savastano S, Falco A, Renzullo A, et al. Preliminary results demonstrating the impact of Mediterranean diet on bone health. J Transl Med. 2017;15(1):1-8.

10. Fernández-Real JM, Bulló M, Moreno-Navarrete JM, Ricart W, Ros E, Estruch R, et al. A mediterranean diet enriched with olive oil is associated with higher serum total osteocalcin levels in elderly men at high cardiovascular risk. J Clin Endocrinol Metab. 2012;97(10):3792-8.

11. Rigacci S, Stefani M. Nutraceutical properties of olive oil polyphenols. An itinerary from cultured cells through animal models to humans. Int $\mathrm{J}$ Mol Sci. 2016;17(6):1-28

12. Bulotta S, Celano M, Lepore SM, Montalcini T, Pujia A, Russo D. Beneficial effects of the olive oil phenolic components oleuropein and hydroxytyrosol: Focus on protection against cardiovascular and metabolic diseases. J Trans Med. 2014;12(1):1-9 
13. Ghanbari R, Anwar F, Alkharfy KM, Gilani AH, Saari N. Valuable nutrients and functional bioactives in different parts of olive (Olea europaea L.): A review. Int J Mol Sci. 2012;13(3):1291-340.

14. Chin KY, Ima-Nirwana S. Olives and bone: A green osteoporosis prevention option. Int J Environ Res Public Health. 2016;13(8):1-11.

15. Summers R, Macnab R. Thyroid, parathyroid hormones and calcium homeostasis. Anaesth Intensive Care Med. 2017;18(10):522-6.

16. Cheung WW, Zhan JY, Paik KH, Mak RH. The impact of inflammation on bone mass in children. Pediatr Nephrol. 2011;26(11):1937-46.

17. Raucci A, Bellosta P, Grassi R, Basilico C, Mansukhani A. Osteoblast proliferation or differentiation is regulated by relative strengths of opposing signaling pathways J Cell Physiol. 2008;215(2):442-51.

18. KomoriT. Regulation of Osteoblast Differentiation by Runx2. Adv Exp Med Biol. 2009;658:43-9.

19. Hong SHH, Lu X, Nanes MS, Mitchell J. Regulation of osterix (Osx, Sp7) and the Osx promoter by parathyroid hormone in osteoblasts. J Mol Endocrinol. 2009;43(5):197-207.

20. Lefebvre V, Bhattaram P. Vertebrate skeletogenesis. Curr Top Dev Biol. 2010;90(C):291-317.

21. Rutkovskiy A, Stensløkken KO, Vaage IJ. Osteoblast differentiation at a glance. Med Sci Monit Basic Res. 2016;22:95-106.

22. Dallas SL, Bonewald LF. Dynamics of the transition from osteoblast to osteocyte Ann NY Acad Sci. 2010;1192(816):437-43.

23. Udagawa N, Takahashi N, AkatsuT, Tanaka H, Sasaki T, NishiharaT, et al. Origin of osteoclasts: mature monocytes and macrophages are capable of differentiating into osteoclasts under a suitable microenvironment prepared by bone marrowderived stromal cells. Proc Natl Acad Sci. 1990;87(18):7260-4.

24. Dolan LC, Hofman-Heather H, Amann N. Hydroxytyrosol: Lack of clastogenicity in a bone marrow chromosome aberration study in rats. BMC Res Notes. 2014;7(1):4-8.

25. Boyce BF, Xing L. Functions of RANKL/RANK/OPG in bone modeling and remodeling. Arch Biochem Biophys. 2008;473(2):139-46.

26. Raggatt LJ, Partridge NC. Cellular and molecular mechanisms of bone remodeling J Biol Chem. 2010;285(33):25103-8.

27. Tagliaferri C, Davicco MJ, Lebecque P, Georgé S, Amiot MJ, Mercier S, et al. Olive oil and vitamin D synergistically prevent bone loss in mice. PLoS One. 2014;9(12):1-19.

28. Small RE. Uses and limitations of bone mineral density measurements in the management of osteoporosis. Medscape Gen Med. 2005;7(2):3.

29. Chen H, Zhou X, Fujita H, Onozuka M, Kubo K. Age-Related Changes in Trabecular and Cortical Bone Microstructure. 2013.

30. Donnelly E. Methods for Assessing Bone Quality. Clin Orthop Relat Res. 2011;469:2128-38

31. Eastell R, Hannon RA. Biomarkers of bone health and osteoporosis risk. Proc Nutr Soc [Internet]. 2008;67(02):157-62. [cited 2018 Aug 15] Available from: http://www.ncbi.nlm.nih.gov/pubmed/18412989

32. Cicerale $S$, Lucas $L$, Keast R. Biological activities of phenolic compounds present in virgin olive oil. Int J Mol Sci. 2010;11(2):458-79.

33. Chen L, Wen $Y$, Kuo C, Chen K. Calcium and vitamin D supplementation on bone health : Current evidence and recommendations. Int J Gerontol. 2014;8(4):183-8.

34. Byberg L, Bellavia A, Larsson SC, Orsini N, Wolk A, Michaëlsson K. Mediterranean Diet and Hip Fracture in Swedish Men and Women. J Bone Miner Res.
2016;31(12):2098-105

35. Mazzanti L, Battino M, Nanetti L, Raffaelli F Alidori A Sforza G, et al. Effect of 1-year dietary supplementation with vitaminized olive oil on markers of bone turnover and oxidative stress in healthy post-menopausal women. Endocrine. 2015;50(2):326-34.

36. Vazquez-Martin A, Fernández-Arroyo S, Cufí S, Oliveras-Ferraros C, Lozano-Sánchez J, Vellón L, et al. Phenolic Secoiridoids in Extra Virgin Olive Oil Impede Fibrogenic and Oncogenic Epithelial-to-Mesenchymal Transition: Extra Virgin Olive Oil As a Source of Novel Antiaging Phytochemicals. Rejuvenation Res [Internet]. 2012;15(1):3-21. Available from: http://online.liebertpub.com/doi/ abs/10.1089/rej.2011.1203

37. García-Martínez O, Mazzaglia G, Sánchez-Ortiz A, Ocaña-Peinado FM, Rivas A. Phenolic content of Sicilian virgin olive oils and their effect on MG-63 human osteoblastic cell proliferation. Grasas Y Aceites. 2014;65(3):e032.

38. Bullon $P$, Battino M, Varela-Lopez A, Perez-Lopez P, Granados-Principal $S$, Ramirez-Tortosa MC, et al. Diets Based on Virgin Olive Oil or Fish Oil but Not on Sunflower Oil Prevent Age-Related Alveolar Bone Resorption by MitochondrialRelated Mechanisms. PLoS One. 2013;8(9):1-11.

39. Puel C, Mardon J, Kati-Coulibaly S, Davicco MJ, Lebecque P, Obled C, et al. Black Lucques olives prevented bone loss caused by ovariectomy and talc granulomatosis in rats. Br J Nutr. 2007;97(5):1012-20.

40. Liu H, Huang $H$, Li B, Wu D, Wang F, Zheng $X$, et al. Olive oil in the prevention and treatment of osteoporosis after artificial menopause. Clin Interv Aging. 2014;4(9):2087-95.

41. Saleh NK, Saleh HA. Olive Oil effectively mitigates ovariectomy-induced osteoporosis in rats. BMC Complement Altern Med [Internet]. 2011;11(1):10. Available from: http://www.biomedcentral.com/1472-6882/11/10

42. Puel C, Quintin A, Agalias A, Mathey J, Obled C, Mazur A, et al. Olive oil and its main phenolic micronutrient (oleuropein) prevent inflammation-induced bone loss in the ovariectomised rat. Br J Nutr [Internet]. 2004;92(01):119. Available from: http://www.journals.cambridge.org/abstract_S0007114504001370

43. Puel C, Mathey J, Agalias A, Kati-coulibaly S, Mardon J, Obled C, et al. Doseresponse study of effect of oleuropein, an olive oil polyphenol, in an ovariectomy/inflammation experimental model of bone loss in the rat. Clin Nutr. 2006;25(5):85968

44. Santiago-Mora R, Casado-Díaz A, De Castro MD, Quesada-Gómez JM. Oleuropein enhances osteoblastogenesis and inhibits adipogenesis: The effect on differentiation in stem cells derived from bone marrow. Osteoporos Int. 2011;22(2):675-84

45. Casado-Díaz A, Anter J, Müller S, Winter P, Quesada-Gómez JM, Dorado G. Transcriptomic analyses of the anti-adipogenic effects of oleuropein in human mesenchymal stem cells. Food Funct. 2017;8(3):1254-70.

46. García-Martínez O, De Luna-Bertos E, Ramos-Torrecillas J, Ruiz C, Milia E, Lorenzo ML, et al. Phenolic compounds in extra virgin olive oil stimulate human osteoblastic cell proliferation. PLoS One. 2016;11(3):1-15

47. Melguizo-Rodriguez L, Manzano-Moreno FJ, De Luna-Bertos E, Rivas A, Ramos-Torrecillas J, Ruiz C, et al. Effect of olive oil phenolic compounds on osteoblast differentiation. Eur J Clin Invest. 2018:48(4):e12904.

48. Casado-Diaz A, Tunez-Finana I, Mata-Granados JM, Ruiz-Mendez MV, Dorado G, Romero-Sanchez MC, et al. Serum from postmenopausal women treated with a by-product of olive-oil extraction process stimulates osteoblastogenesis and inhibits adipogenesis in human mesenchymal stem-cells (MSC). Exp Gerontol. 2017;90:71-8

\section{GRAPHICAL ABSTRACT}

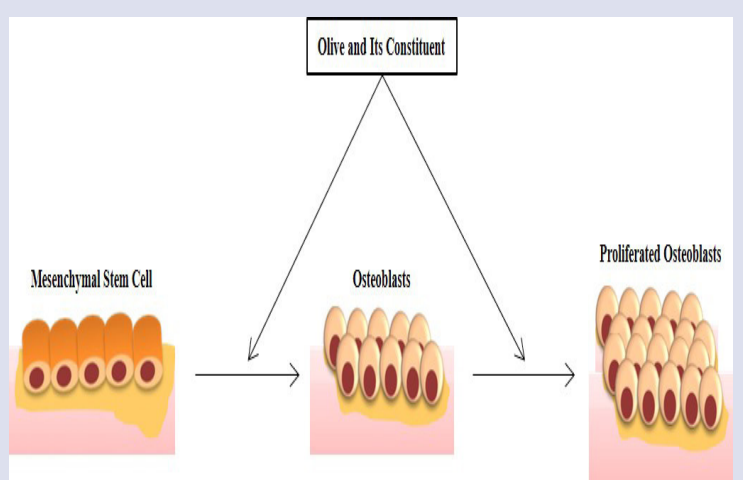

\section{SUMMARY}

- Bone homeostasis is the balance between bone resorption and bone formation that is regulated by the plethora of signaling molecules including transcription factors, cytokines and surface receptor proteins. Association of Mediterranean diet and bone health has been the subject of interest among scientists in recent years. Olive and its constituents have been suggested to be the major contributor to the benefits of the Mediterranean diet in bone health. Many studies ranging from clinical, animal and in vitro cell culture has reported benefits of olive and its constituents in bone health. In this review, cellular and molecular mechanism of bone homeostasis and the influence of olive and its constituents were discussed. Olive and its constituent exerted its beneficial effect in bone health through stimulation of bone formation as opposed to inhibition of bone resorption. Specifically, olive and its constituent has been reported to promote osteoblast differentiation and proliferation.

Cite this article: Idrus RBH, Saim AB. Olea europaea and its Constituents Promote Bone Health by Enhancing Osteoblast Differentiation and Proliferation: A Review. Pharmacog J. 2019;11(1):01-07. 